Journal of Mathematics and Statistics 6 (4): 409-415, 2010

ISSN 1549-3644

(C) 2010 Science Publications

\title{
Smooth Neighborhood Structures in a Smooth Topological Spaces
}

\author{
A.A. Ramadan and M.A. Abdel-Sattar \\ Department of Mathematics, Faculty of Science, \\ Beni-Suef University, Beni-Suef, Egypt
}

\begin{abstract}
Problem Statement: Various concepts related to a smooth topological spaces have been introduced and relations among them studied by several authors (Chattopadhyay, Ramadan, etc). Conclusion/Recommendations: In this study, we presented the notions of three sorts of neighborhood structures of a smooth topological spaces and give some of their properties which are results by Ying extended to smooth topological spaces.
\end{abstract}

Key words: Fuzzy smooth topology, smooth neighborhood structures

\section{INTRODUCTION}

Šostak (1985) introduced the fuzzy topology as an extension of Chang (1968) fuzzy topology. It has been developed in many directions (Ramadan, 1992; Chattopadhyay and Samanta, 1993; EL Gayyar et al., 1994; Höhle and Rodabaugh, 1998; Kubiak and Šostak, 1997; Demirici, 1997; Ramadan et al., 2001; 2009; Abdel-Sattar, 2006).

Ying (1994) studied the theory of neighborhood systems in fuzzy topology with the method used to develop fuzzifying topology (Ying, 1991) by treating the membership relation as a fuzzy relation. In this study, we generate the structures of neighborhood systems in a smooth topology with the method used in (Ying, 1991), by using fuzzy sets and fuzzy points.

Notions and preliminaries: The class of all fuzzy sets on a universal set $\mathrm{X}$ will be denote by $\mathrm{L}^{\mathrm{X}}$, where $\mathrm{L}$ is the special lattice and $\mathrm{L}=([0,1], \leq)$. Also, $\mathrm{L}_{0}=(0,1]$ and $\mathrm{L}_{1}=[0,1)$.

Definition 1: $\mathrm{Pu}$ and Liu (1980) a fuzzy set in $\mathrm{X}$ is called a fuzzy point iff it takes the value 0 for all $y \in X$, except one, say $\mathrm{x} \in \mathrm{X}$. If its value at $\mathrm{x}$ is $\lambda(0<\lambda \leq 1)$ we denote this fuzzy point by $\mathrm{x}_{\lambda}$, where the point $\mathrm{x}$ is called its support. The fuzzy point is said to be contained in a fuzzy set $A$, or belong to $A$, denoted by $\mathrm{x}_{\lambda} \in \mathrm{A}$, iff $\lambda \leq \mathrm{A}(\mathrm{x})$. Evidently, every fuzzy set $\mathrm{A}$ can be expressed as the union of all fuzzy points which belong to $\mathrm{A}$.

Definition 2: Ying (1991) Let $\mathrm{X}$ be a non-empty set. Let $\mathrm{x}_{\lambda}$ be a fuzzy point in $\mathrm{X}$ and let $\mathrm{A}$ be a fuzzy subset of $\mathrm{X}$. Then the degree to which $\mathrm{x}_{\lambda}$ belongs to $\mathrm{A}$ is:

$$
\mathrm{m}\left(\mathrm{x}_{\lambda}, \cup_{\mathrm{i} \in \Gamma} \mathrm{A}_{\mathrm{i}}\right)=\mathrm{V}_{\mathrm{i} \in \Gamma} \mathrm{m}\left(\mathrm{x}_{\lambda}, \mathrm{A}_{\mathrm{i}}\right)
$$

Obviously, we have the following properties:

(1) $\mathrm{m}(\mathrm{x}, \mathrm{A})=\mathrm{A}(\mathrm{x})$

(2) $\mathrm{m}\left(\mathrm{x}_{\lambda}, \mathrm{A}\right)=1$ iff $\mathrm{x}_{\lambda} \in \mathrm{A}, \mathrm{m}\left(\mathrm{x}_{\lambda}, \mathrm{A}\right)=0$ iff $\lambda=1$ and $\mathrm{A}(\mathrm{x})=0$

(3) $\mathrm{m}\left(\mathrm{x}_{\lambda}, \mathrm{E}_{\mathrm{i} \hat{\Gamma} \Gamma} \mathrm{A}_{\mathrm{i}}\right)=\mathrm{U}_{\mathrm{i} \hat{\Gamma} \Gamma} \mathrm{m}\left(\mathrm{x}_{\lambda}, \mathrm{A}_{\mathrm{i}}\right)$, (generalized multiple choice principles)

Definition 3: Ying (1991) let $(X, \tau)$ be a fuzzy topological space (fts, for short), let e be a fuzzy point in $X$ and let $A$ be a fuzzy subset of $X$. Then the degree to which $\mathrm{A}$ is a neighborhood of $\mathrm{e}$ is defined by:

$$
\mathrm{N}_{\mathrm{e}}(\mathrm{A})=\sup \{\mathrm{m}(\mathrm{e}, \mathrm{B}): \mathrm{B} \in \tau, \mathrm{B} \subseteq \mathrm{A}\}
$$

Thus $\mathrm{N}_{\mathrm{e}} \in \mathrm{L}^{\mathrm{L}^{\mathrm{X}}}$ is called the fuzzy neighborhood system of e in $(X, \tau)$.

Definition 4: Ying (1991) let $(X, \tau)$ be a fts, e a fuzzy point in $\mathrm{X}$ and $\mathrm{A}$ a fuzzy subset of $\mathrm{X}$.

Then the degree to which $\mathrm{e}$ is an adherent point of A is given as:

$$
\operatorname{ad}(e, A)=\inf _{B \subseteq A^{c}}\left(1-N_{e}(B)\right)
$$

where, $\mathrm{A}^{\mathrm{c}}$ is the complement of $\mathrm{A}$.

Definition 5: Ramadan (1992) A smooth topological space (sts, for short) is an ordered pair $(X, \tau)$, where $X$ is a non-empty set and $\tau: \mathrm{L}^{\mathrm{X}} \rightarrow \mathrm{L}$ is a mapping satisfying the following properties:

Corresponding Author: A.A. Ramadan, Department of Mathematics, Faculty of Science, Beni-Suef University, Beni-Suef, Egypt 
(O1) $\tau(\underline{1})=\tau(\underline{0})=1$

(O2) For all $\mathrm{A}_{1}, \mathrm{~A}_{2}, \in \mathrm{L}^{\mathrm{X}}, \tau\left(\mathrm{A}_{1} \cap \mathrm{A}_{2}\right) \geq \tau\left(\mathrm{A}_{1}\right) \wedge \tau\left(\mathrm{A}_{2}\right)$

(O3) $\forall \mathrm{I}, \tau\left(\cup_{\mathrm{i} \in \Gamma} \mathrm{A}_{\mathrm{i}}\right) \geq \wedge_{\mathrm{i} \in \Gamma} \tau\left(\mathrm{A}_{\mathrm{i}}\right)$

Definition 6: EL Gayyar et al. (1994) let $(\mathrm{X}, \tau)$ be a sts and $\alpha \in \mathrm{L}_{\mathrm{o}}$. Then the family: $\tau_{\alpha}=\left\{\mathrm{A} \in \mathrm{L}^{\mathrm{X}}: \tau(\mathrm{A}) \geq \alpha\right\}$. which is clearly a fuzzy topology Chang (1968) sense.

Definition 7: Demirici (1997) Let $(\mathrm{X}, \tau)$ be a sts and $\mathrm{A} \in \mathrm{L}^{\mathrm{X}}$. Then the $\tau$-smooth interior of $\mathrm{A}$, denoted by:

$$
\mathrm{A}^{0}=\bigcup\left\{\mathrm{B} \in \mathrm{L}^{\mathrm{X}}: \tau(\mathrm{B})>0, \mathrm{~B} \subseteq \mathrm{A}\right\}
$$

Remark 1: Demirici (1997) let $\tau$ be a Chang's fuzzy topology (CFT, for short) on the non-empty set X. Then the smooth topology and smooth cotopology $\tau_{\mathrm{s}}, \tau_{\mathrm{s}}{ }^{*}$ : $\mathrm{L}^{\mathrm{X}} \rightarrow \mathrm{L}$, defined by:

$$
\tau(\mathrm{A})= \begin{cases}1, & \text { if } \mathrm{A} \in \tau \\ 0, & \text { if } \mathrm{A} \notin \tau\end{cases}
$$

and $\tau_{\mathrm{s}}^{*}(\mathrm{~A})=\tau\left(\mathrm{A}^{\mathrm{c}}\right)$ for each $\mathrm{A} \in \mathrm{L}^{\mathrm{X}}$, identify the CFT $\tau$ and corresponding fuzzy cotopology for it. Thus the $\tau_{\mathrm{s}}$ smooth interior of $\mathrm{A}$ is:

$$
\begin{aligned}
\mathrm{A}^{0} & =\bigcup\left\{\mathrm{B} \in \mathrm{L}^{\mathrm{x}}: \tau_{\mathrm{s}}(\mathrm{B})>0, \mathrm{~B} \subseteq \mathrm{A}\right\} \\
& =\bigcup\left\{\mathrm{B} \in \mathrm{L}^{\mathrm{X}}: \mathrm{B} \in \tau, \mathrm{B} \subseteq \mathrm{A}\right\}
\end{aligned}
$$

This show that $\mathrm{A}^{\mathrm{o}}$ is exactly the interior of $\mathrm{A}$ with respect to $\tau$ in Chang (1968) sense.

Lemma 1: Ramadan (1992) $\sup _{\alpha \in L} \sup \{A(x) \wedge B(x)$ : $\mathrm{A}(\mathrm{x}) \geq \alpha\}=\sup _{\alpha \in \mathrm{L}} \sup \{\alpha \wedge \mathrm{B}(\mathrm{x}): \mathrm{A}(\mathrm{x}) \geq \alpha\}$.

Smooth neighborhood systems of a fuzzy set: Here, we build a smooth neighborhood systems of a fuzzy set in a sts and we give some of its properties.

For a mapping $\mathrm{M}: \mathrm{L}^{\mathrm{X}} \rightarrow \mathrm{L}^{\mathrm{LX}}$ and $\mathrm{A} \in \mathrm{L}^{\mathrm{X}}, \alpha \in[0 ; 1)$; let us define the family $\mathrm{M}_{\mathrm{A}}^{\alpha}=\left\{\mathrm{B} \in \mathrm{L}^{\mathrm{X}}: \mathrm{M}_{\mathrm{A}}(\mathrm{B})>\alpha\right\}$; which will play an important role in this part.

Definition 8: Let $(X, \tau)$ be a sts and $A \in L^{X}$ : Then a mapping $\mathrm{N}_{\mathrm{A}}: \mathrm{L}^{\mathrm{X}} \rightarrow \mathrm{L}^{\mathrm{L}^{\mathrm{X}}}$ is called the smooth neighborhood (nbd, for short) of A with respect to the st $\tau$ iff for each $\alpha \in[0,1)$ :

$$
\mathrm{N}_{\mathrm{A}}^{\alpha}=\left\{\mathrm{B} \in \mathrm{L}^{\mathrm{X}}:\left(\exists \mathrm{C} \in \tau^{\alpha}\right)(\mathrm{A} \subseteq \mathrm{C} \subseteq \mathrm{B})\right\}
$$

where, $\tau^{\alpha}=\left\{A \in L^{X}: \tau(A)>\alpha\right\}$ the strong $\alpha$ - level of $\tau$.

\section{Remark 2:}

- The real number $\mathrm{N}_{A}(B)$ is called the degree of nbdness of the fuzzy set B to the fuzzy set A. If the smooth nbd system of a fuzzy set $\mathrm{A}$ has the following property: $\mathrm{N}_{\mathrm{A}}\left(\mathrm{L}^{\mathrm{X}}\right) \subseteq\{0,1\}$, then $\mathrm{N}_{\mathrm{A}}$ is called the fuzzy nbd system of $A$

- We say that the family $\left(\mathrm{N}_{\mathrm{A}}\right)_{\alpha}=\left\{\mathrm{B}: \mathrm{N}_{\mathrm{A}}(\mathrm{B})>\alpha\right\}$ is a fuzzy nbd system of $A$ for each $\alpha \in[0,1)$ and $\left(\mathrm{N}_{\mathrm{A}}\right)_{\alpha}$ is called the strong $\alpha$-level fuzzy nbd of A

Proposition 1: Let $(X, \tau)$ be a sts and $A \in L^{X}$. Then a mapping $\mathrm{N}_{\mathrm{A}}: \mathrm{L}^{\mathrm{X}} \rightarrow \mathrm{L}^{\mathrm{LX}}$ is the smooth nbd system of $\mathrm{A}$ with respect to the st $\tau$ iff:

$$
\mathrm{N}_{\mathrm{A}}(\mathrm{B}) \begin{cases}\sup \{\tau(\mathrm{C}): \mathrm{A} \subseteq \mathrm{C} \subseteq \mathrm{B}\}, & \text { if } \mathrm{A} \subseteq \mathrm{B} \\ 0, & \text { if } \mathrm{A} \nsubseteq \mathrm{B}\end{cases}
$$

\section{Proof:}

(1) Suppose that the mapping $\mathrm{N}_{\mathrm{A}}$ : $\mathrm{L}^{\mathrm{X}} \rightarrow \mathrm{L}^{\mathrm{LX}}$ is the smooth nbd systems of A with respect to the st $\tau$. Consider the following two cases:

- For the case $A \not \subset B$, suppose that $N_{A}(B)>0$. From Definition 1, there exists $C \in \tau^{\alpha}$ such that $\mathrm{A} \subseteq \mathrm{C} \subseteq \mathrm{B}$, i.e., $A \subseteq B$, a contradiction. Thus $\mathrm{N}_{\mathrm{A}}(\mathrm{B})=0$

- For the case $A \subseteq B$. We may have $N_{A}(B)=0$ or $\mathrm{N}_{\mathrm{A}}(\mathrm{B})>0$. If $\mathrm{N}_{\mathrm{A}}(\mathrm{B})=0$, then it is obvious that $\mathrm{N}_{\mathrm{A}}(\mathrm{B})=0 \leq \sup \{\tau(\mathrm{C}): \mathrm{A} \subseteq \mathrm{C} \subseteq \mathrm{B}\}$, if $\sup \{\tau(\mathrm{C})$ : $\mathrm{A} \subseteq \mathrm{C} \subseteq \mathrm{B}\}=\lambda>0$, then $\exists \mathrm{C} \in \mathrm{L}^{\mathrm{X}}$ such that $\tau(\mathrm{C})>0$ and $\mathrm{A} \subseteq \mathrm{C} \subseteq \mathrm{B}$ : We obtain $\mathrm{N}_{\mathrm{A}}(\mathrm{B})>0$, a contradiction

Therefore:

$$
\mathrm{N}_{\mathrm{A}}(\mathrm{B})=0=\sup \{\tau(\mathrm{C}): \mathrm{A} \subseteq \mathrm{C} \subseteq \mathrm{B}\}
$$

Now suppose that $\mathrm{N}_{\mathrm{A}}(\mathrm{B})=\lambda>0$. For an arbitrary $0<\varepsilon \leq \lambda$, we have $N_{A}(B)=\lambda$ - $\varepsilon$, i.e., $B \in N_{A}^{\lambda-\varepsilon}$. Since the mapping: $\mathrm{N}_{\mathrm{A}}: \mathrm{L}^{\mathrm{X}} \rightarrow \mathrm{L}^{\mathrm{LX}}$ is a smooth nbd system of $\mathrm{A}, \exists C \in \mathrm{L}^{\mathrm{X}}$ such that $\mathrm{C} \in \tau^{\lambda-\varepsilon}$ and $\mathrm{A} \subseteq \mathrm{C} \subseteq \mathrm{B}$, i.e., $\sup \{\tau(C): A \subseteq C \subseteq B\}>\lambda-\varepsilon$. Since $\varepsilon>0$ is arbitrary we have:

$$
\sup \{\tau(C): A \subseteq C \subseteq B\} \geq \lambda=N_{A}(B)
$$

On the other hand, let $\sup \{\tau(\mathrm{C}): \mathrm{A} \subseteq \mathrm{C} \subseteq \mathrm{B}\}=\gamma>0$. Then for every $0<\varepsilon \leq \gamma, \exists C \in L^{X}$ such that $\tau(C)>\gamma-\varepsilon$ and $A \subseteq C \subseteq B$. Therefore $B \in N_{A}^{\gamma-\varepsilon}$, i.e., $N_{A}(B)>\gamma-\varepsilon$. Since $\varepsilon$ is an arbitrary we have: 


$$
\mathrm{N}_{\mathrm{A}}(\mathrm{B}) \geq \gamma=\sup \{\tau(\mathrm{C}): \mathrm{A} \subseteq \mathrm{C} \subseteq \mathrm{B}\}
$$

Hence the inequality follows:

(2) For $\alpha \in[0,1)$, let $\mathrm{B} \in N_{A}^{\alpha}$, i.e., $\mathrm{N}_{\mathrm{A}}(\mathrm{B})>\alpha$ : Then we can write $\alpha<\mathrm{N}_{\mathrm{A}}(\mathrm{B})=\sup \{\tau(\mathrm{C})$ : $\mathrm{A} \subseteq \mathrm{C} \subseteq \mathrm{B}\}$,i.e., $\exists \mathrm{C} \in \mathrm{L}^{\mathrm{X}}$ such that $\tau(\mathrm{C})>\alpha, \mathrm{A} \subseteq \mathrm{C} \subseteq \mathrm{B}$. Then we have:

$$
\mathrm{N}_{\mathrm{A}}^{\alpha} \subseteq\left\{\mathrm{B} \in \mathrm{L}^{\mathrm{X}}:\left(\exists \mathrm{C} \in \tau^{\alpha}\right)(\mathrm{A} \subseteq \mathrm{C} \subseteq \mathrm{B})\right\}
$$

By the same way we can show that:

$$
\left\{\mathrm{B} \in \mathrm{L}^{\mathrm{X}}:\left(\exists \mathrm{C} \in \tau^{\alpha}\right)(\mathrm{A} \subseteq \mathrm{C} \subseteq \mathrm{B})\right\} \subseteq \mathrm{N}_{\mathrm{A}}^{\alpha}
$$

Hence:

$$
\mathrm{N}_{\mathrm{A}}^{\alpha}=\left\{\mathrm{B} \in \mathrm{L}^{\mathrm{X}}:\left(\exists \mathrm{C} \in \tau^{\alpha}\right)(\mathrm{A} \subseteq \mathrm{C} \subseteq \mathrm{B})\right\}
$$

Remark 3: In Proposition 3, the fuzzy subsets $A$ of $X$ can be replaced by the fuzzy points on $\mathrm{X}$, that is, by the special fuzzy subsets e, in this case:

$$
\mathrm{N}_{\mathrm{e}}(\mathrm{A}) \begin{cases}\sup \{\tau(\mathrm{C}): \mathrm{e} \in \mathrm{C} \subseteq \mathrm{A}\}, & \text { if } \mathrm{e} \in \mathrm{A} \\ 0, & \text { if } \mathrm{e} \notin \mathrm{A}\end{cases}
$$

Proposition 2: Let $(X, \tau)$ be a sts and $A \in \mathrm{L}^{\mathrm{X}}$. If the mapping $\mathrm{N}_{\mathrm{A}}: \mathrm{L}^{\mathrm{X}} \rightarrow \mathrm{L}^{\mathrm{L}^{\mathrm{X}}}$ is the smooth nbd system of $A$ with respect to the st $\tau$, then the following properties hold:

$$
\begin{aligned}
& \text { (N1) } \mathrm{N}_{\underline{0}}(\underline{0})=\mathrm{N}_{\underline{1}}(\underline{1})=1 \text { and } \mathrm{N}_{\mathrm{A}}(\mathrm{B})>0 \Rightarrow \mathrm{A} \subseteq \mathrm{B} \\
& \text { (N2) If } \mathrm{A}_{1} \subseteq \mathrm{A} \text { and } \mathrm{B} \subseteq \mathrm{B}_{1} \text {, then } \mathrm{N}_{\mathrm{A}}(\mathrm{B}) \leq \mathrm{N}_{\mathrm{A}_{1}}\left(\mathrm{~B}_{1}\right) \\
& \text { (N3) } \mathrm{N}_{\mathrm{A}}\left(\mathrm{B}_{1}\right) \wedge \mathrm{N}_{\mathrm{A}}\left(\mathrm{B}_{2}\right) \leq \mathrm{N}_{\mathrm{A}}\left(\mathrm{B}_{1} \cap \mathrm{B}_{2}\right) \\
& \text { (N4) } \mathrm{N}_{\mathrm{A}}(\mathrm{B}) \leq \sup _{\mathrm{A} \subseteq \mathrm{C} \subseteq \mathrm{B}}\left\{\mathrm{N}_{\mathrm{A}}(\mathrm{C}) \wedge \mathrm{N}_{\mathrm{C}}(\mathrm{B})\right\}, \forall \mathrm{A}, \mathrm{B}, \mathrm{C} \in \mathrm{L}^{\mathrm{X}}
\end{aligned}
$$

Proof: (N1) and (N2) follows directly from Definition 1 and Proposition 3. (N3) Suppose that $\mathrm{N}_{\mathrm{A}}\left(\mathrm{B}_{1}\right)=\alpha_{1}>0$ and $\mathrm{N}_{\mathrm{A}}\left(\mathrm{B}_{2}\right)>\alpha_{2}>0$. Then for a fixed $\varepsilon>0$ such that: $\varepsilon \leq$ $\alpha_{1} \wedge \alpha_{2} \Rightarrow N_{A}\left(B_{1}\right)>\alpha_{1}-\varepsilon \geq 0$ and $N_{A}\left(B_{2}\right)>\alpha_{2}-\varepsilon \geq 0$. From Definition 1, it is clear that there exists $\mathrm{C}_{1}, \mathrm{C}_{2} \in \mathrm{L}^{\mathrm{X}}$ such that:

$$
\begin{aligned}
& \tau\left(\mathrm{C}_{1}\right)>\alpha_{1}-\varepsilon, \tau\left(\mathrm{C}_{2}\right)>\alpha_{2}-\varepsilon \text { and } \\
& \mathrm{A} \subseteq \mathrm{C}_{1} \subseteq \mathrm{B}_{1}, \mathrm{~A} \subseteq \mathrm{C}_{2} \subseteq \mathrm{B}_{2}
\end{aligned}
$$

Therefore, $\tau\left(\mathrm{C}_{1} \cap \mathrm{C}_{2}\right) \geq \tau\left(\mathrm{C}_{1}\right) \wedge \tau\left(\mathrm{C}_{2}\right)>\left(\alpha_{1}-\varepsilon\right) \wedge\left(\alpha_{2}-\varepsilon\right)$ $=\left(\alpha_{1} \wedge \alpha_{2}\right)-\varepsilon$ and $A \subseteq C_{1} \cap C_{2} \subseteq B_{1} \cap B_{2}$. Thus $\mathrm{N}_{\mathrm{A}}\left(\mathrm{B}_{1} \cap \mathrm{B}_{2}\right) \geq\left(\alpha_{1} \wedge \alpha_{2}\right)-\varepsilon$ : Since $\varepsilon$ is arbitrary, we find that $\mathrm{N}_{\mathrm{A}}\left(\mathrm{B}_{1} \cap \mathrm{B}_{2}\right) \geq \mathrm{N}_{\mathrm{A}}\left(\mathrm{B}_{1}\right) \wedge \mathrm{N}_{\mathrm{A}}\left(\mathrm{B}_{2}\right)$. (N4) $\mathrm{N}_{\mathrm{A}}(\mathrm{B})=\sup \{\tau(\mathrm{C})$ : $A \subseteq C \subseteq B$ \}. From Proposition 3, we obtain $\tau(C) \leq N_{A}(C)$ and $\tau(C) \leq N_{C}(B)$. Hence:

Thus, $\sup \{\tau(C): A \subseteq C \subseteq B\} \leq \sup \left\{N_{A}(C) \wedge N_{C}(B)\right\}$.

$$
\mathrm{N}_{\mathrm{A}}(\mathrm{B}) \leq \sup _{\mathrm{A} \subseteq \mathrm{C} \subseteq \mathrm{B}}\left\{\mathrm{N}_{\mathrm{A}}(\mathrm{C}) \wedge \mathrm{N}_{\mathrm{C}}(\mathrm{B})\right\}
$$

Smooth neighborhood systems of a fuzzy points: Definition 9: Let $(X, \tau)$ be a sts, e a fuzzy point in $X$ and $A$ be a fuzzy subset of $X$.

Then the degree to which $\mathrm{A}$ is a NBD of $e$ is defined by:

$$
\mathrm{N}_{\mathrm{e}}(\mathrm{A})=\left\{\begin{array}{lc}
\sup _{\mathrm{B} \subseteq \mathrm{A}}\{\mathrm{m}(\mathrm{e}, \mathrm{B}) \wedge \tau(\mathrm{B}): \tau(\mathrm{B})>0\} & \text { if } \mathrm{m}(\mathrm{e}, \mathrm{A})>0 \\
0, & \text { otherwise }
\end{array}\right.
$$

Thus $\mathrm{N}_{\mathrm{e}} \in \mathrm{L}^{\mathrm{L}^{\mathrm{X}}}$ is called the smooth NBD system of $\mathrm{e}$ in $(\mathrm{X}, \tau)$.

Remark 4: It is clear that when a fuzzy point $e \in$ $\mathrm{B} \in \mathrm{L}^{\mathrm{X}}$, then $\mathrm{m}(\mathrm{e}, \mathrm{B})=1$ and

$$
N_{e}(A)=\left\{\begin{array}{lr}
\sup _{B \subseteq A}\{\tau(B): e \in B \subseteq A\}, & \text { if } e \in A \\
0, & \text { if } \mathrm{e} \notin A
\end{array}\right.
$$

is the NBD systems in the sense of Demirici (1997)

Remark 5: For any crisp point $\mathrm{x}$ in $\mathrm{X}$, we have:

$$
\mathrm{N}_{\mathrm{x}}(\mathrm{A})=\sup _{\mathrm{B} \subseteq \mathrm{A}}\{\mathrm{B}(\mathrm{x}) \wedge \tau(\mathrm{B}): \tau(\mathrm{B})>0\}, \mathrm{B}(\mathrm{x}) \neq 0 .
$$

Proposition 3: The NBD systems $\mathrm{N}_{\mathrm{e}}$ of e in sts can be constructed from the cuts $\tau_{\alpha}, \alpha \in(0,1]$, by using the equality:

$$
\mathrm{N}_{\mathrm{e}}(\mathrm{A})=\sup _{\alpha>0}\left\{\left[\mathrm{~N}_{\mathrm{e}}^{*}(\mathrm{~A})\right]^{\alpha} \wedge \alpha\right\}
$$

where, $\left[\mathrm{N}_{\mathrm{e}}^{*}(\mathrm{~A})\right]^{\alpha}=\sup \left\{\mathrm{m}(\mathrm{e}, \mathrm{B}): \mathrm{B} \subseteq \mathrm{A}, \mathrm{B} \in \tau_{\alpha}\right\}$, is the NBD systems in the sense of (Ying, 1994; Theorem 1).

Proof: By using Definition 9, we have:

$$
\begin{aligned}
\mathrm{N}_{\mathrm{e}}(\mathrm{A}) & =\sup _{\mathrm{B} \subseteq \mathrm{A}}\{\mathrm{m}(\mathrm{e}, \mathrm{B}) \wedge \tau(\mathrm{B}): \tau(\mathrm{B})>0\} \\
& =\sup _{\alpha>0} \sup _{\mathrm{B} \subseteq \mathrm{A}}\{\mathrm{m}(\mathrm{e}, \mathrm{B}) \wedge \alpha: \tau(\mathrm{B}) \geq \alpha\} \\
& =\sup _{\alpha>0}\left\{\sup _{\mathrm{B} \subseteq \mathrm{A}}\{\mathrm{B}(\mathrm{x}): \tau(\mathrm{B}) \geq \alpha\} \wedge \alpha\right\} \\
& =\sup _{\alpha>0}\left\{\sup _{\mathrm{B} \subseteq \mathrm{A}}\left\{\mathrm{m}(\mathrm{e}, \mathrm{B}): \mathrm{B} \in \tau_{\alpha}\right\} \wedge \alpha\right\} \\
& =\sup _{\alpha>0}\left\{\left[\mathrm{~N}_{\mathrm{e}}^{*}(\mathrm{~A})\right]^{\alpha} \wedge \alpha\right\}
\end{aligned}
$$


Remark 6: For any crisp point $\mathrm{x}$ in $\mathrm{X}$; we have:

$$
\mathrm{N}_{\mathrm{x}}(\mathrm{A})=\sup _{\alpha>0}\left\{\left[\mathrm{~N}_{\mathrm{x}}^{*}(\mathrm{~A})\right]^{\alpha} \wedge \alpha\right\}
$$

where, $\left[\mathrm{N}_{\mathrm{x}}^{*}(\mathrm{~A})\right]^{\alpha}=\sup _{\mathrm{B} \subseteq \mathrm{A}}\left\{\mathrm{B}(\mathrm{x}): \mathrm{B} \in \tau_{\alpha}\right\}$.

Theorem 1: Let $(X, \tau)$ be a sts and e a fuzzy point of $X$. If the mapping $\mathrm{N}_{\mathrm{e}}: \mathrm{L}^{\mathrm{X}} \rightarrow \mathrm{L}$ is the smooth NBD systems of $\mathrm{e}$ with respect to $\tau$, then the following properties hold:

$$
\begin{aligned}
& \text { (N1) } N_{e}(A) \leq m(e, B) \\
& \text { (N2) If } A \subseteq B \text { and } A, B \in L^{x}, \text { then } N_{e}(A) \leq N_{e}(B) \\
& \text { (N3) For all } A, B \in L^{x}, N_{e}(A \cap B) \geq N_{e}(A) \wedge N_{e}(B) \\
& (N 4) N_{e}(A) \leq \sup _{\alpha>0}\left\{\operatorname { s u p } _ { B \subseteq A } \left\{\left[N_{e}^{*}(B)^{\alpha} \wedge \alpha:\right.\right.\right.
\end{aligned}
$$$$
\text { for all fuzzy point } \left.\left.\mathrm{d}, \mathrm{m}(\mathrm{d}, \mathrm{B}) \leq \mathrm{N}_{\mathrm{d}}^{*}(\mathrm{~B})\right\}\right\}
$$

Proof: (N1) and (N2) follows directly from Remark 2. (N3):

$$
\begin{aligned}
& \min \left(\mathrm{N}_{\mathrm{e}}(\mathrm{A}), \mathrm{N}_{\mathrm{e}}(\mathrm{B})\right) \\
& =\min \left(\sup _{\mathrm{C} \subseteq \mathrm{A}}\{\mathrm{m}(\mathrm{e}, \mathrm{C}) \wedge \tau(\mathrm{C}): \tau(\mathrm{C})>0\}, \sup _{\mathrm{D} \subseteq \mathrm{B}}\right. \\
& \{\mathrm{m}(\mathrm{e}, \mathrm{D}) \wedge \tau(\mathrm{D}): \tau(\mathrm{D})>0\}) \\
& =\sup _{\mathrm{D} \subseteq \mathrm{B}} \sup _{\mathrm{C} \subseteq \mathrm{A}}\{\min (\mathrm{m}(\mathrm{e}, \mathrm{C}), \mathrm{m}(\mathrm{e}, \mathrm{D})) \wedge \tau(\mathrm{C}) \wedge \tau(\mathrm{D}): \\
& \tau(\mathrm{C}), \tau(\mathrm{D})>0\} \\
& =\sup _{\mathrm{D}_{\subseteq \mathrm{B}}} \sup _{\mathrm{C} \subseteq \mathrm{A}}\{\min (\mathrm{m}(\mathrm{e}, \mathrm{C} \cap \mathrm{D}) \wedge \tau(\mathrm{C}) \wedge \tau(\mathrm{D}): \tau(\mathrm{C}), \\
& \tau(\mathrm{D})>0\} \\
& \leq \sup _{\mathrm{D} \subseteq \mathrm{B}} \sup _{\mathrm{C} \subseteq \mathrm{A}}\{\min (\mathrm{m}(\mathrm{e}, \mathrm{C} \cap \mathrm{D}) \wedge \tau(\mathrm{C} \cap \mathrm{D}): \\
& \tau(\mathrm{C}), \tau(\mathrm{D})>0\} \\
& \leq \sup _{\mathrm{E} \subseteq \mathrm{A} \cap \mathrm{B}}\{(\mathrm{m}(\mathrm{e}, \mathrm{E}) \wedge \tau(\mathrm{E}): \tau(\mathrm{E})>0, \mathrm{C} \cap \mathrm{D}=\mathrm{E}\} \\
& =\mathrm{N}_{\mathrm{e}}(\mathrm{A} \cap \mathrm{B})
\end{aligned}
$$

(N4) Combining axiom (4) in Theorem 1, in (Ying, 1994) and Proposition 4, (N4) follows.

Theorem 2: Let the mapping $\mathrm{N}_{\mathrm{e}}$ : $\mathrm{L}^{\mathrm{X}} \rightarrow \mathrm{L}$ satisfy the conditions (N1)-(N4), then the mapping $\tau: \mathrm{L}^{\mathrm{X}} \rightarrow \mathrm{L}$ defined by:

$$
\tau(\mathrm{A})= \begin{cases}\inf _{\mathrm{e}}\left\{\mathrm{m}(\mathrm{e}, \mathrm{A}) \wedge \mathrm{N}_{\mathrm{e}}(\mathrm{A})\right\}, & \text { if } \mathrm{m}(\mathrm{e}, \mathrm{A})>0, \mathrm{~A} \neq \underline{0} \text { and } \mathrm{A} \neq \underline{1} \\ 1, & \text { if } \mathrm{A}=\underline{0} \text { or } \mathrm{A}=\underline{1}\end{cases}
$$

Where:

$A \in L^{X}=A$ st on $X$, furthermore the mapping

$\mathrm{N}_{\mathrm{e}} \quad$ = Exactly the smooth nbd systems of e with respect to st $\tau$.

Proof: (O1) Obvious.
$(\mathrm{O} 2)$ :

$$
\begin{aligned}
\tau(\mathrm{A} \cap \mathrm{B}) & =\inf _{\mathrm{e}}\left\{\mathrm{m}(\mathrm{e}, \mathrm{A} \cap \mathrm{B}) \wedge \mathrm{N}_{\mathrm{e}}(\mathrm{A} \cap \mathrm{B})\right\} \\
& \geq \inf _{\mathrm{e}}\left\{\mathrm{m}(\mathrm{e}, \mathrm{A} \cap \mathrm{B}) \wedge\left(\min \left(\mathrm{N}_{\mathrm{e}}(\mathrm{A}), \mathrm{N}_{\mathrm{e}}(\mathrm{B})\right)\right)\right\} \\
& =\inf _{\mathrm{e}}\left\{\min (\mathrm{m}(\mathrm{e}, \mathrm{A}), \mathrm{m}(\mathrm{e}, \mathrm{B})) \wedge\left(\min \left(\mathrm{N}_{\mathrm{e}}(\mathrm{A}), \mathrm{N}_{\mathrm{e}}(\mathrm{B})\right)\right)\right\} \\
& =\min \left\{\inf _{\mathrm{e}}\left\{\mathrm{m}(\mathrm{e}, \mathrm{A}) \wedge \mathrm{N}_{\mathrm{e}}(\mathrm{A})\right\}, \inf _{\mathrm{e}}\left\{\mathrm{m}(\mathrm{e}, \mathrm{B}) \wedge \mathrm{N}_{\mathrm{e}}(\mathrm{B})\right\}\right) \\
& =\min (\tau(\mathrm{A}), \tau(\mathrm{B}))
\end{aligned}
$$

(O3):

$$
\begin{aligned}
\tau\left(\bigcup_{i \in I} A_{i}\right) & =\inf _{e}\left\{m\left(e, \bigcup_{i \in I} A_{i}\right) \wedge N_{e}\left(\bigcup_{i \in I} A_{i}\right)\right\} \\
& =\inf _{e}\left\{\sup _{i \in I} m\left(e, A_{i}\right) \wedge N_{e}\left(\bigcup_{i \in I} A_{i}\right)\right\} \\
& \geq \inf _{e}\left\{\sup _{i \in I} m\left(e, A_{i}\right) \wedge \inf _{i \in I} N_{e}\left(A_{i}\right)\right\} \\
& \geq \inf _{e}\left\{\inf _{i \in I} m\left(e, A_{i}\right) \wedge \inf _{i \in I} N_{e}\left(A_{i}\right)\right\} \\
& =\inf _{i \in I} \inf _{e}\left\{m\left(e, A_{i}\right) \wedge N_{e}\left(A_{i}\right)\right\} \\
& =\inf _{i \in I} \tau\left(A_{i}\right)
\end{aligned}
$$

Now, we show that the mapping $\mathrm{N}_{\mathrm{e}}$ : $\mathrm{L}^{\mathrm{X}} \rightarrow \mathrm{L}$ which satisfies the conditions (N1)-(N4) is exactly the smooth NBD systems of e for the sts $(X, \tau)$ : Let the mapping $M_{e}: L^{X} \rightarrow L$ be the smooth NBD systems of e of the sts $(\mathrm{X}, \tau)$. Then applying $\left(\mathrm{N}_{1}\right)$ we have:

$$
\begin{aligned}
M_{e}(A) & =\sup _{B \subseteq A}\{m(e, B) \wedge \tau(B)\} \\
& =\sup _{B \subseteq A}\left\{m(e, B) \wedge \inf _{p}\left(m(p, B) \wedge N_{p}(B)\right)\right\}
\end{aligned}
$$

Since:

$$
\inf _{p}\left(m(p, B) \wedge N_{p}(B)\right)=\inf _{p} m(p, B) \wedge \inf _{p} N_{p}(B) \leq \inf _{p} N_{p}(B) \leq N_{e}(B)
$$

Thus:

$$
\mathrm{M}_{\mathrm{e}}(\mathrm{A}) \leq \sup _{\mathrm{B} \subseteq \mathrm{A}}\left\{\mathrm{m}(\mathrm{e}, \mathrm{B}) \wedge \mathrm{N}_{\mathrm{e}}(\mathrm{B})\right\} \leq \mathrm{N}_{\mathrm{e}}(\mathrm{A})
$$

On the other hand, using (N4) and Theorem 1, in (Ying, 1994) we may write:

$N_{\mathrm{x}_{\lambda}}(\mathrm{A}) \leq \sup _{\alpha>0}\left\{\sup _{\mathrm{B} \subseteq \mathrm{A}}\left\{\left[\mathrm{N}_{\mathrm{x}_{\lambda}}^{*}(\mathrm{~B})\right]^{\alpha} \wedge \alpha\right.\right.$ :for

each fuzzy point $\left.\left.d, m(e, B) \leq N_{d}^{*}(B)\right\}\right]$

$$
\leq \sup _{\alpha>0}\left\{\operatorname { s u p } _ { \mathrm { B } \subseteq \mathrm { A } } \left\{\left[\mathrm{~N}_{\mathrm{x}_{\lambda}}^{*}(\mathrm{~B})\right]^{\alpha} \wedge \alpha\right.\right. \text { :for each }
$$

crisp point $\left.\left.\mathrm{y}, \mathrm{m}(\mathrm{y}, \mathrm{B}) \leq \mathrm{N}_{\mathrm{y}}^{*}(\mathrm{~B})\right\}\right]$

$$
\begin{aligned}
& =\sup _{\alpha>0}\left\{\sup _{\mathrm{B} \subseteq \mathrm{A}}\left\{\left[\mathrm{N}_{\mathrm{x}_{\lambda}}^{*}(\mathrm{~B})\right]^{\alpha} \wedge \alpha: \mathrm{B} \in \tau_{\alpha}\right\}\right\} \\
& =\sup _{\alpha>0}\left\{\operatorname { s u p } _ { \mathrm { B } \subseteq \mathrm { A } } \left\{\min \left(1,1-\lambda+\mathrm{N}_{\mathrm{x}}^{*}(\mathrm{~B})\right) \wedge \alpha:\right.\right.
\end{aligned}
$$

$\left.\left.\mathrm{B} \in \tau_{\alpha}\right\}\right\}$

$$
\leq \sup _{\alpha>0}\left\{\sup _{\mathrm{B} \subseteq \mathrm{A}}\{\min (1,1-\lambda+\mathrm{m}(\mathrm{x}, \mathrm{B})) \wedge \alpha:\right.
$$

$\left.\left.\mathrm{B} \in \tau_{\alpha}\right\}\right\}$

$$
\begin{aligned}
& =\sup _{\alpha>0}\left\{\sup _{\mathrm{B} \subseteq \mathrm{A}}\left\{\mathrm{m}\left(\mathrm{x}_{\lambda}, \mathrm{B}\right) \wedge \alpha: \mathrm{B} \in \tau_{\alpha}\right\}\right\} \\
& =\sup _{\mathrm{B} \subseteq \mathrm{A}}\left\{\mathrm{m}\left(\mathrm{x}_{\lambda}, \mathrm{B}\right) \wedge \tau(\mathrm{B})\right\} \\
& =\mathrm{M}_{\mathrm{x}_{\lambda}}(\mathrm{A})
\end{aligned}
$$


Hence, the equality $N_{e}=M_{e}$ follows at once from (1) and (2).

Definition 10: Let $(X, \tau)$ be a sts, e a fuzzy point in $X$ and A a fuzzy subset of $X$. Then the degree to which $\mathrm{e}$ is an adherent point of $\mathrm{A}$ is given as:

$$
\operatorname{ad}(e, A)=\inf _{B \subseteq A^{c}}\left(1-N_{e}(B)\right.
$$

where, $\mathrm{A}^{\mathrm{c}}$ is the complement of $\mathrm{A}$.

Remark 6: For any crisp point $\mathrm{x}$ in $\mathrm{X}$, we have:

$$
\operatorname{ad}(x, A)=\inf _{B \subseteq A^{c}}\left(1-N_{x}(B)\right)
$$

\section{Proposition 4:}

$$
\begin{aligned}
& \operatorname{ad}(e, A)=\inf _{\alpha>0}\left\{[\operatorname{ad}(e, A)]^{\alpha} \vee(1-\alpha)\right\} \\
& {[\operatorname{ad}(e, A)]^{\alpha}=\inf _{B \subseteq A^{c}}\left(1-\left[N_{e}^{*}(B)\right]^{\alpha}\right)}
\end{aligned}
$$

Proof: Follows from Proposition 4.

\section{Proposition 5:}

$$
\left.\mathrm{N}_{\mathrm{x}_{\lambda}}(\mathrm{A}) \leq \sup _{\alpha>0}\left\{\min \left(1,1-\lambda+\left[\mathrm{N}_{\mathrm{x}}^{*}(\mathrm{~A})^{\alpha}\right]\right) \wedge \alpha\right)\right\}
$$

\section{Proof:}

$$
\begin{aligned}
\mathrm{N}_{\mathrm{x}_{\lambda}}(\mathrm{A}) & =\sup _{\alpha>0}\left\{\left[\mathrm{~N}_{\mathrm{x}_{\lambda}}^{*}(\mathrm{~A})\right]^{\alpha} \wedge \alpha\right\} \\
& =\sup _{\alpha>0}\left\{\sup _{\mathrm{B} \subseteq \mathrm{A}}\left\{\mathrm{m}\left(\mathrm{x}_{\lambda}, \mathrm{B}\right): \mathrm{B} \in \tau_{\alpha}\right\} \wedge \alpha\right\} \\
& =\sup _{\alpha>0}\left\{\sup _{\mathrm{B} \subseteq \mathrm{A}}\left\{\min (1,1-\lambda+\mathrm{m}(\mathrm{x}, \mathrm{B})): \mathrm{B} \in \tau_{\alpha}\right\} \wedge \alpha\right\} \\
& \leq \sup _{\alpha>0}\left\{\min \left(1,1-\lambda+\sup _{\mathrm{B} \subseteq \mathrm{A}}\left\{\mathrm{m}(\mathrm{x}, \mathrm{B}): \mathrm{B} \in \tau_{\alpha}\right\}\right) \wedge \alpha\right\} \\
& =\sup _{\alpha>0}\left\{\min \left(1,1-\lambda+\left[\mathrm{N}_{\mathrm{x}}^{*},(\mathrm{~A})\right]^{\alpha}\right) \wedge \alpha\right\}
\end{aligned}
$$

Fuzzy smooth r-neighborhood:

Definition 11: Let $(\mathrm{X}, \tau)$ be a sts, $\mathrm{A} \in \mathrm{L}^{\mathrm{X}}$, e a fuzzy point in $X$ and $r \in L_{0}$. Then the degree to which $A$ is a fuzzy smooth $\mathrm{r}$-nbd system of e is defined by:

$$
\mathrm{N}_{\mathrm{e}}(\mathrm{A}, \mathrm{r})=\sup _{\mathrm{B} \subseteq \mathrm{A}}\{\mathrm{m}(\mathrm{e}, \mathrm{B}): \tau(\mathrm{B}) \geq \mathrm{r}\}
$$

A mapping $\mathrm{N}_{\mathrm{e}}$ : $\mathrm{L}^{\mathrm{X}} \times \mathrm{L}_{0} \rightarrow \mathrm{L}$ is called the fuzzy smooth r-nbd system of e.

Theorem 2: Let $(X, \tau)$ be a sts and $N_{e}$ the fuzzy smooth $r$-nbd system of e. For $A, B \in L^{X}$ and $r, s \in L_{0}$, it satisfies the following properties:
(1) $\mathrm{N}_{\mathrm{e}}(\mathrm{A}, \mathrm{r}) \leq \mathrm{m}(\mathrm{e}, \mathrm{A})$ for each $\mathrm{r} \in \mathrm{L}_{0}$

(2) $\mathrm{N}_{\mathrm{e}}(\mathrm{A}, \mathrm{r}) \leq \mathrm{N}_{\mathrm{e}}(\mathrm{B}, \mathrm{r})$, if $\mathrm{A} \subseteq \mathrm{B}$

(3) $\mathrm{N}_{\mathrm{e}}(\mathrm{A}, \mathrm{r}) \wedge \mathrm{N}_{\mathrm{e}}(\mathrm{B}, \mathrm{r}) \leq \mathrm{N}_{\mathrm{e}}(\mathrm{A} \cap \mathrm{B}, \mathrm{r})$

(4) $\mathrm{N}_{\mathrm{e}}(\mathrm{A}, \mathrm{r}) \leq \sup \left\{\mathrm{N}_{\mathrm{e}}(\mathrm{B}, \mathrm{r}): \mathrm{B} \leq \mathrm{A}, \mathrm{m}(\mathrm{d}, \mathrm{B}) \leq \mathrm{N}_{\mathrm{d}}(\mathrm{B}, \mathrm{r})\right.$; for all fuzzy point $\mathrm{d}$ in $\mathrm{X}$ \}

(5) $\mathrm{N}_{\mathrm{e}}(\mathrm{A}, \mathrm{r}) \geq \mathrm{N}_{\mathrm{e}}(\mathrm{A}, \mathrm{s})$, if $\mathrm{r} \leq \mathrm{s}$

(6) $\mathrm{N}_{\mathrm{x}_{\mathrm{t}}}(\mathrm{A}, \mathrm{r})=\min \left(1,1-\mathrm{t}+\mathrm{N}_{\mathrm{x}_{1}}(\mathrm{~A}, \mathrm{r})\right)$

Proof: (2) and (5) are easily proved.

(1) It is proved from the following:

$$
\begin{aligned}
\mathrm{N}_{\mathrm{e}}(\mathrm{A}, \mathrm{r}) & =\sup \left\{\mathrm{m}\left(\mathrm{e}, \mathrm{B}_{\mathrm{i}}\right): \mathrm{B}_{\mathrm{i}} \leq \mathrm{A}, \tau\left(\mathrm{B}_{\mathrm{i}}\right) \geq \mathrm{r}\right\} \\
& =\left\{\mathrm{m}\left(\mathrm{e}, \cup \mathrm{B}_{\mathrm{i}}\right): \cup \mathrm{B}_{\mathrm{i}} \leq \mathrm{A}, \tau\left(\cup \mathrm{B}_{\mathrm{i}}\right) \geq \mathrm{r}\right\} \\
& \leq \mathrm{m}(\mathrm{e}, \mathrm{A})
\end{aligned}
$$

Suppose there exist $\mathrm{A}, \mathrm{B} \in \mathrm{L}^{\mathrm{X}}$ and $\mathrm{r} \in \mathrm{L}_{0}$ such that:

$$
N_{e}(A, r) \wedge N_{e}(B, r)>t>N_{e}(A \cap B, r)
$$

Since $N_{e}(A, r)>t$ and $N_{e}(B, r)>t$, there exist $\mathrm{C}_{1}, \mathrm{C}_{2} \in \mathrm{L}^{\mathrm{X}}$ with:

$$
\mathrm{C}_{1} \subseteq \mathrm{A}, \tau\left(\mathrm{C}_{1}\right) \geq \mathrm{r}, \mathrm{C}_{2} \subseteq \mathrm{B}, \tau\left(\mathrm{C}_{2}\right) \geq \mathrm{r}
$$

Such that:

$$
\mathrm{m}\left(\mathrm{e}, \mathrm{C}_{1}\right) \wedge \mathrm{m}\left(\mathrm{e}, \mathrm{C}_{2}\right)=\mathrm{m}\left(\mathrm{e}, \mathrm{C}_{1} \cap \mathrm{C}_{2}\right)>\mathrm{t}
$$

On the other hand, since:

$$
\mathrm{C}_{1} \cap \mathrm{C}_{2} \subseteq \mathrm{A} \cap \mathrm{B}, \tau\left(\mathrm{C}_{1} \cap \mathrm{C}_{2}\right) \geq \mathrm{r}
$$

We have:

$$
\mathrm{N}_{\mathrm{e}}(\mathrm{A} \cap \mathrm{B}, \mathrm{r}) \geq \mathrm{m}\left(\mathrm{e}, \mathrm{C}_{1} \cap \mathrm{C}_{2}\right)>\mathrm{t}
$$

It is a contradiction.

(4) If $\tau(B) \geq r$, then $N_{d}(B, r)=m(d, B)$; for each fuzzy point $d$ in $X$. It implies:

$$
\begin{aligned}
& \mathrm{N}_{\mathrm{e}}(\mathrm{A}, \mathrm{r}) \\
& =\sup \{\mathrm{m}(\mathrm{e}, \mathrm{B}): \mathrm{B} \subseteq \mathrm{A}, \tau(\mathrm{B}) \geq \mathrm{r}\} \\
& =\sup \left\{\mathrm{N}_{\mathrm{e}}(\mathrm{B}, \mathrm{r}): \mathrm{B} \subseteq \mathrm{A}, \mathrm{N}_{\mathrm{d}}(\mathrm{B}, \mathrm{r})=\mathrm{m}(\mathrm{d}, \mathrm{B}),\right. \text { for all } \\
& \text { fuzzy point } \mathrm{d} \text { in } \mathrm{X}\} \\
& \leq \sup \left\{\mathrm{N}_{\mathrm{e}}(\mathrm{B}, \mathrm{r}): \mathrm{B} \subseteq \mathrm{A}, \mathrm{m}(\mathrm{d}, \mathrm{B}) \leq \mathrm{N}_{\mathrm{d}}(\mathrm{B}, \mathrm{r})\right. \text {, for all } \\
& \text { fuzzy point } \mathrm{d} \text { in } \mathrm{X}\}
\end{aligned}
$$

(6) It proved from: 


$$
\begin{aligned}
\mathrm{N}_{\mathrm{x}_{\mathrm{t}}}(\mathrm{A}, \mathrm{r}) & =\sup \left\{\mathrm{m}\left(\mathrm{x}_{\mathrm{t}}, \mathrm{B}\right): \mathrm{B} \subseteq \mathrm{A}, \tau(\mathrm{B}) \geq \mathrm{r}\right\} \\
& =\sup \{\min (1,1-\mathrm{t}+\mathrm{B}(\mathrm{x})): \mathrm{B} \subseteq \mathrm{A}, \tau(\mathrm{B}) \geq \mathrm{r}\} \\
& =\min (1,1-\mathrm{t}+\sup \{\mathrm{B}(\mathrm{x}): \mathrm{B} \subseteq \mathrm{A}, \tau(\mathrm{B}) \geq \mathrm{r}\}) \\
& =\min \left(1,1-\mathrm{t}+\mathrm{N}_{\mathrm{x}}(\mathrm{A}, \mathrm{r})\right)
\end{aligned}
$$

Theorem 3: Let $\mathrm{N}_{e}$ be the fuzzy smooth r-nbd system of e satisfying the above conditions (1)-(5), the function $\tau_{\mathrm{N}}: \mathrm{L}^{\mathrm{X}} \rightarrow \mathrm{L}$ defined by:

$$
\begin{aligned}
& \tau_{\mathrm{N}}(\mathrm{A})=\mathrm{V}\left\{\mathrm{r} \in \mathrm{L}_{0}: \mathrm{m}(\mathrm{e}, \mathrm{A})=\mathrm{N}_{\mathrm{e}}(\mathrm{A}, \mathrm{r})\right. \text { for all } \\
& \text { fuzzy point } \mathrm{e} \text { in } \mathrm{X}\}
\end{aligned}
$$

has the following properties:

(1) $\tau_{\mathrm{N}}$ is a st. on $X$

(2) If $\mathrm{N}_{\mathrm{e}}$ is the fuzzy nbd systems of e induced by $(\mathrm{X}, \tau)$, then $\tau_{\mathrm{N}}=\tau$

(3) If $\mathrm{N}_{\mathrm{e}}$ satisfy the conditions (1)-(6), then:

$$
\tau_{\mathrm{N}}(\mathrm{A})=\mathrm{V}\left\{\mathrm{r} \in \mathrm{L}_{0}: \mathrm{m}(\mathrm{x}, \mathrm{A})=\mathrm{N}_{\mathrm{x}}(\mathrm{A}, \mathrm{r}), \forall \mathrm{x} \in \mathrm{X}\right\}
$$

Proof: (1) We will show that $\tau_{N}\left(B_{1} \cap B_{2}\right) \geq \tau_{N}\left(B_{1}\right) \wedge$ $\tau_{N}\left(B_{2}\right)$, for any $B_{1}, B_{2} \in L^{X}$.

Suppose there exist $\mathrm{B}_{1}, \mathrm{~B}_{2} \in \mathrm{L}^{\mathrm{X}}$ and $\mathrm{r} \in \mathrm{L}_{0}$ such that:

$$
\tau_{\mathrm{N}}\left(\mathrm{B}_{1} \cap \mathrm{B}_{2}\right)<\mathrm{r}<\tau_{\mathrm{N}}\left(\mathrm{B}_{1}\right) \wedge \tau_{\mathrm{N}}\left(\mathrm{B}_{2}\right)
$$

For each $i \in\{1,2\}$ there exists $r_{i} \in L_{0}$ with: $\mathrm{m}\left(\mathrm{e}, \mathrm{B}_{\mathrm{i}}\right)=\mathrm{N}_{\mathrm{e}}\left(\mathrm{B}_{\mathrm{i}}, \mathrm{r}_{\mathrm{i}}\right)$; for all fuzzy point e in $\mathrm{X}$

Such that: $\tau_{N}\left(B_{i}\right) \geq r_{i}>r$,

From (I), (II) and (5), we have:

$\mathrm{m}\left(\mathrm{e}, \mathrm{B}_{\mathrm{i}}\right)=\mathrm{N}_{\mathrm{e}}\left(\mathrm{B}_{\mathrm{i}}, \mathrm{r}_{\mathrm{i}}\right) \leq \mathrm{N}_{\mathrm{e}}\left(\mathrm{B}_{\mathrm{i}} \mathrm{r}\right) \leq \mathrm{m}\left(\mathrm{e}, \mathrm{B}_{\mathrm{i}}\right)$

It implies $\mathrm{m}\left(\mathrm{e}, \mathrm{B}_{\mathrm{i}}\right)=\mathrm{N}_{\mathrm{e}}\left(\mathrm{B}_{\mathrm{i}}, \mathrm{r}\right)$ : Furthermore:

$$
\begin{aligned}
\mathrm{m}\left(\mathrm{e}, \mathrm{B}_{1} \cap \mathrm{B}_{2}\right) & =\mathrm{N}_{\mathrm{e}}\left(\mathrm{B}_{1}, \mathrm{r}\right) \wedge \mathrm{N}_{\mathrm{e}}\left(\mathrm{B}_{2}, \mathrm{r}\right) \\
& \leq \mathrm{N}_{\mathrm{e}}\left(\mathrm{B}_{1} \cap \mathrm{B}_{2}, \mathrm{r}\right) \\
& \leq \mathrm{m}\left(\mathrm{e}, \mathrm{B}_{1} \cap \mathrm{B}_{2}\right)
\end{aligned}
$$

Thus, $\quad \mathrm{N}_{\mathrm{e}}\left(\mathrm{B}_{1} \cap \mathrm{B}_{2}, \quad \mathrm{r}\right) \quad=\quad \mathrm{m}\left(\mathrm{e}, \mathrm{B}_{1} \cap \mathrm{B}_{2}\right)$, i.e., $\tau N\left(B_{1} \cap B_{2}\right) \geq r$. It is a contradiction for the Eq. I.

Suppose there exists $B=\cup_{i \in \Gamma} B_{i} \in L_{X}$ and $r_{0} \in L_{0}$ such that:

$$
\tau_{\mathrm{N}}(\mathrm{B})<\mathrm{r}_{0}<\widehat{\mathrm{i} \in \Gamma}_{\mathrm{N}} \tau_{\mathrm{N}}\left(\mathrm{B}_{\mathrm{i}}\right)
$$

For each $i \in \Gamma$, there exists $r_{i} \in L_{0}$ with $\mathrm{m}\left(\mathrm{e}, \mathrm{B}_{\mathrm{i}}\right)=\mathrm{N}_{\mathrm{e}}\left(\mathrm{B}_{\mathrm{i}}, \mathrm{r}_{\mathrm{i}}\right)$; for all fuzzy point $\mathrm{e}$ in $\mathrm{X}$

Such that: $\tau_{\mathrm{N}}\left(\mathrm{B}_{\mathrm{i}}\right) \geq \mathrm{r}_{\mathrm{i}}>\mathrm{r}$

From (I), (IV) and (5), we have:

$$
\mathrm{m}\left(\mathrm{e}, \mathrm{B}_{\mathrm{i}}\right)=\mathrm{N}_{\mathrm{e}}\left(\mathrm{B}_{\mathrm{i}}, \mathrm{r}_{\mathrm{i}}\right) \leq \mathrm{N}_{\mathrm{e}}\left(\mathrm{B}_{\mathrm{i}}, \mathrm{r}\right) \leq \mathrm{m}\left(\mathrm{e}, \mathrm{B}_{\mathrm{i}}\right)
$$

It implies $\mathrm{m}\left(\mathrm{e}, \mathrm{B}_{\mathrm{i}}\right)=\mathrm{N}_{\mathrm{e}}\left(\mathrm{B}_{\mathrm{i}}, \mathrm{r}\right)$ : Furthermore:

$$
\begin{aligned}
\mathrm{m}\left(\mathrm{e}, \cup_{\mathrm{i} \in \Gamma} \mathrm{B}_{\mathrm{i}}\right) & =\mathrm{V}_{\mathrm{i} \in \Gamma} \mathrm{m}\left(\mathrm{e}, \mathrm{B}_{\mathrm{i}}\right) \\
& =\mathrm{V}_{\mathrm{i} \in \Gamma} \mathrm{N}_{\mathrm{e}}\left(\mathrm{B}_{\mathrm{i}}, \mathrm{r}_{\mathrm{i}}\right) \\
& \leq \mathrm{N}_{\mathrm{e}}\left(\cup_{\mathrm{i} \in \Gamma} \mathrm{B}_{\mathrm{i}}, \mathrm{r}\right) \\
& \leq \mathrm{m}\left(\mathrm{e}, \cup_{\mathrm{i} \in \Gamma} \mathrm{B}_{\mathrm{i}}\right) .
\end{aligned}
$$

Thus, $\mathrm{N}_{\mathrm{e}}\left(\cup_{\mathrm{i} \in \Gamma} \quad \mathrm{B}_{\mathrm{i}}, \quad r\right)=\mathrm{m}\left(\mathrm{e}, \cup_{\mathrm{i} \in \Gamma} \mathrm{B}_{\mathrm{i}}\right)$, i.e., $\tau_{N}\left(\cup_{i \in \Gamma} B_{i}\right) \geq r_{0}$. It is a contradiction for the Eq. III.

(2) Suppose there exists $A \in L^{X}$ such that:

$$
\tau_{\mathrm{N}}(\mathrm{A})>\tau(\mathrm{A})
$$

From the Definition of $\tau_{\mathrm{N}}$, there exists $\mathrm{r}_{0} \in \mathrm{L}_{0}$ with $\mathrm{m}(\mathrm{e}, \mathrm{A})=\mathrm{N}_{\mathrm{e}}\left(\mathrm{A}, \mathrm{r}_{0}\right)$ such that:

$$
\tau_{\mathrm{N}}(\mathrm{A}) \geq \mathrm{r}_{0}>\tau(\mathrm{A})
$$

Since:

$\mathrm{m}(\mathrm{e}, \mathrm{A})=\mathrm{N}_{\mathrm{e}}\left(\mathrm{A}, \mathrm{r}_{0}\right)=\sup \left\{\mathrm{m}\left(\mathrm{e}, \mathrm{B}_{\mathrm{i}}\right): \mathrm{B}_{\mathrm{i}} \subseteq \mathrm{A}, \tau\left(\mathrm{B}_{\mathrm{i}}\right) \geq \mathrm{r}_{0}\right\}$

Then, for each $\mathrm{x} \in \mathrm{X}$ :

$$
\left(\cup \mathrm{B}_{\mathrm{i}}\right)(\mathrm{x})=\sup \left\{\mathrm{m}\left(\mathrm{x}, \mathrm{B}_{\mathrm{i}}\right): \mathrm{B}_{\mathrm{i}} \subseteq \mathrm{A}\right\}=\mathrm{m}(\mathrm{x}, \mathrm{A})=\mathrm{A}(\mathrm{x})
$$

Thus, $A=\cup B_{i} . S_{o}, \tau(A) \geq r_{0}$. It is a contradiction. Suppose there exists $A \in L^{X}$ such that:

$$
\tau_{\mathrm{N}}(\mathrm{A})<\tau(\mathrm{A})
$$

There exists $\mathrm{r}_{1} \in \mathrm{L}_{0}$ such that:

$$
\tau_{\mathrm{N}}(\mathrm{A})<\mathrm{r}_{1} \leq \tau(\mathrm{A})
$$

Since $\tau(A) \geq r_{1}$, we have:

$$
\mathrm{N}_{\mathrm{e}}\left(\mathrm{A}, \mathrm{r}_{1}\right)=\sup \left\{\mathrm{m}(\mathrm{e}, \mathrm{B}): \mathrm{B} \subseteq \mathrm{A}, \tau(\mathrm{B}) \geq \mathrm{r}_{1}\right\}=\mathrm{m}(\mathrm{e}, \mathrm{A})
$$


Hence $\tau_{N}(A) \geq r_{1}$. It is a contradiction.

\section{CONCLUSION}

(3) We only show that $m\left(x_{t}, A\right)=N_{x}(A, r)$, for all fuzzy point $x_{t}$ in $X$ iff $m(x, A)=A(x)=N_{x}(A, r), \forall x \in$ $\mathrm{X}$ :

$(\Rightarrow)$ It is trivial.

$(\Leftarrow)$ From the condition $(6)$ :

$$
\begin{aligned}
\mathrm{N}_{\mathrm{x}_{\mathrm{t}}}(\mathrm{A}, \mathrm{r}) & =\min \left(1,1-\mathrm{t}+\mathrm{N}_{\mathrm{x}}(\mathrm{A}, \mathrm{r})\right) \\
& =\min (1,1-\mathrm{t}+\mathrm{m}(\mathrm{x}, \mathrm{A})) \\
& =\min (1,1-\mathrm{t}+\mathrm{A}(\mathrm{x})) \\
& =\mathrm{m}\left(\mathrm{x}_{\mathrm{t}}, \mathrm{A}\right) .
\end{aligned}
$$

Example 1: Let $X=\{a, b\}$ be a set, $N$ a natural number set and $\mathrm{B} \in \mathrm{L}^{\mathrm{X}}$ as follows:

$$
\mathrm{B}(\mathrm{a})=0.3, \quad \mathrm{~B}(\mathrm{~b})=0.4
$$

We define a smooth fuzzy topology:

$$
\tau(\mathrm{A})=\left\{\begin{array}{cc}
1, & \text { if } \mathrm{A}=\underline{0} \text { or } \underline{1}, \\
\frac{1}{2}, & \text { if } \quad \mathrm{A}=\mathrm{B}, \\
0, & \text { otherwise }
\end{array}\right.
$$

From Definition $1, \mathrm{~N}_{\mathrm{a}}, \mathrm{N}_{\mathrm{b}}$ : $\mathrm{L}^{\mathrm{X}} \times \mathrm{L}_{0} \rightarrow \mathrm{L}$ as follows:

$$
\begin{aligned}
& \mathrm{N}_{\mathrm{a}}(\mathrm{A})=\left\{\begin{array}{cc}
1, & \text { if } \mathrm{A}=\underline{1}, \quad \mathrm{r} \in \mathrm{L}_{0} \\
0.3, & \text { if } \underline{1} \neq \mathrm{A} \supseteq \mathrm{B}, \quad 0<\mathrm{r} \leq \frac{1}{2} \\
0, & \text { otherwise }
\end{array}\right. \\
& \mathrm{N}_{\mathrm{b}}(\mathrm{A})=\left\{\begin{array}{cc}
1, & \text { if } \mathrm{A}=\underline{1}, \quad \mathrm{r} \in \mathrm{L}_{0} \\
0.4, & \text { if } 1 \neq \mathrm{A} \supseteq \mathrm{B}, \quad 0<\mathrm{r} \leq \frac{1}{2} \\
0, & \text { otherwise }
\end{array}\right.
\end{aligned}
$$

From Theorem 2 and Theorem 3 (3), we have:

$$
\tau_{N}(\mathrm{~A})=\left\{\begin{array}{lc}
1, & \text { if } \mathrm{A}=\underline{0} \text { or } \underline{1}, \\
\frac{1}{2}, & \text { if } \quad \mathrm{A}=\mathrm{B} \\
0, & \text { otherwise }
\end{array}\right.
$$

\section{REFERENCES}

Abdel-Sattar, M.A., 2006. Some structures of L-fuzzy topology. J. Fuzzy Math., 14: 751-766.

Chang, C.L., 1968. Fuzzy topological spaces. J. Math. Anal. Appli., 24: 182-190.

Chattopadhyay, K.C. and S.K. Samanta, 1993. Fuzzy topology: Fuzzy closure operator, fuzzy compactness and fuzzy connectedness. Fuzzy Sets and Syst., 54: 207-212. DOI: 10.1016/01650114(93)90277-O

Demirici, M., 1997. Neighborhood structures of smooth topological spaces. Fuzzy Sets Syst., 92: 123-128. DOI: 10.1016/S0165-0114(96)00132-7

El Gayyar, M.K., E.E. Kerre and A.A. Ramadan, 1994. Almost compactness and near compactness in smooth topological spaces. Fuzzy Sets Syst., 62: 193-202. DOI: 10.1016/0165-0114(94)90059-0

Höhle, U. and S.E. Rodabaugh, 1998. Mathematics of Fuzzy Sets: Logic, Topology and Measure Theory. 1st Edn., Springer, USA., ISBN: 10: 0792383885, pp: 736.

Kubiak, T. and A. Šostak, 1997. Lower set-valued fuzzy topologies. Quaest. Math., 20: 423-429. DOI: 10.1080/16073606.1997.9632016

Pu, P.M. and Y.M. Liu, 1980. Fuzzy topology I: Neighborhood structure of a fuzzy point and Moore-Smith convergence. J. Math. Anal. Appli. 76: 571-599.

Ramadan, A.A., 1992. Smooth topological spaces. Fuzzy Sets Syst., 48: 371-375. DOI: 10.1016/01650114(92)90352-5

Ramadan, A.A., S.N. El-Deeb and M.A. Abdel-Sattar, 2001. On smooth topological spaces IV. Fuzzy Sets Syst., 119: 473-482. DOI: 10.1016/S01650114(99)00083-4

Ramadan, A.A., M.A. Abdel-Sattar and Y.C. Kim, 2009. The fuzzy neighborhood structures. Int. J. Math. Comput., 3: 146-156.

Šostak, A.P., 1985. On a fuzzy topological structures. Suppl. Rend. Circ. Mat Palermo Ser. II, 11: 89-103.

Ying, M., 1991. A new approach for fuzzy topology I. Fuzzy Sets and Syst., 39: 303-321. DOI: 10.1016/0165-0114(91)90100-5

Ying, M., 1994. On the method of neighborhood systems in fuzzy topology. Fuzzy Sets Syst., 68: 227-238. DOI: 10.1016/0165-0114(94)90048-5 